\title{
Dynamic Dynamometry to Characterize Disk Turbines for Space-Based Power
}

\author{
Zhiyuan Yang, Hope L. Weiss, Matthew J. Traum ${ }^{1}$ \\ Mechanical Engineering Department, Milwaukee School Of Engineering, Milwaukee, WI
}

\begin{abstract}
Rankine cycles will someday be the power plants of choice for manned space missions, providing excellent thermodynamic efficiency and high power density. The Rankine cycle's hallmark is a working fluid that changes phase between liquid and vapor. However, the working fluid must remain in the vapor phase as it passes through the turbine to avoid damaging this component. The need to tightly regulate the working fluid phase through the turbine imposes limits on the power produced and the overall efficiency of the cycle, especially given limitations on power plant volume and mass necessarily imposed by housing it in an interplanetary spacecraft.
\end{abstract}

These limitations could be relaxed if a turbine were incorporated into the Rankine power cycle that was robust and fully operational while processing two-phase flows. Disk turbines have the potential for continuous operation regardless of the thermodynamic quality of working fluid running through them. However, due to high rotational velocity and low torque output by disk turbines, their performance is difficult to evaluate using conventional techniques for aero-derived turbines.

To assess disk turbines as candidates for space-based power generation, we describe a method to accurately measure and predict turbine mechanical power output using the rational inertia of the turbine's spinning components and friction in its bearings as the load. The turbine's time response to Dirac load inputs, as well as its no-load responses to compressed air input over a range of pressures, are measured. This technique, called dynamic dynamometry, produces turbine power-versus-angular-velocity curves, useful for quantitative performance analysis.

\section{Introduction}

Working microgravity Rankine cycle technology is identified by NASA as an essential element for future manned space missions to provide high-power density and superior thermodynamic efficiency ${ }^{\mathrm{i}}$ compared to existing space-based energy generation methods. ${ }^{\text {ii }}$ Given the extensive experience NASA has developed to handle fluids, especially low-temperature fluids, in microgravity, a space-based Rankine cycle could be implemented today using existing technology. ${ }^{\text {ii }}$ The hallmark of the Rankine cycle is a working fluid that changes phase between liquid and vapor. However, the working fluid must remain in the vapor phase as it passes through

\footnotetext{
${ }^{1}$ The corresponding author gratefully acknowledges the Wisconsin Space Grant Consortium 2012-2013 Higher Education Incentives Award Program for the financial support it provided to make this project possible. All authors gratefully acknowledge EASENET, Inc. for project financial and in-kind support. This paper's undergraduate lead author is a member of the Milwaukee Undergraduate Researcher Incubator (MURI) at MSOE, an organization which fast-tracks undergraduates into meaningful early research experiences.
} 
the turbine to avoid damage. This necessity imposes limits on the power produced and the overall efficiency of the cycle.

Better performance could be obtained if a non-conventional turbine were incorporated into the Rankine power cycle that operated reliably and robustly while processing two-phase flows. Disk turbines have the potential for continuous operation without damage when functioning with a working fluid of fractional thermodynamic quality. ${ }^{\text {iv }}$ Disk turbines (also called boundary layer turbines or Tesla turbines) differ from conventional aero-derived turbines. Instead of gas impinging on aerodynamic blade surfaces to produce lift and spin the shaft, disk turbines rely on viscous shear between the working fluid and flat disks to provide motive torque. As a result, disk turbines typically operate at much higher rotational rates with lower torque than their aeroderived counterparts, ${ }^{\mathrm{v}}$ which makes their performance difficult to evaluate using conventional techniques for aero-derived turbines. ${ }^{\text {vi }}$

Experimental evaluation of engines and turbo-machinery typically requires a dynamometer to measure power curves - power output as a function of rotational rate for a series of loads. Due to the high-rotation-rate and low torque output by disk turbines, no commercially-available dynamometers are suitable. Instead, we use a technique called dynamic dynamometry, which uses the rotational inertia of the turbine spindle and the friction in the bearings as the load. No separate dynamometer is needed to extract power curves. This technique has already been used successfully by researchers to characterize tiny disk turbines, ${ }^{\text {vii }}$ and its application as a classroom demonstration to teach mechanical engineering concepts has also been described. ${ }^{\text {viii }}$

This paper explains how the dynamic dynamometry technique can be applied to measure the rotational inertia and extract power curves for a disk turbine. This technique provides the foundation for future characterization and analysis of disk turbines for space-based power generation.

\section{Theory and Experiments}

All described experiments were carried out using a small pre-built disk turbine made available by an industry partner (Figure 1). The complete apparatus consisted of an optical tachometer located to enable continuous measurement of the turbine shaft rotation rate and a video recording device (an iPhone). The video-recorded tachometer readout provided time histories of the turbine shaft angular velocity during experimental events, which was the fundamental data stream analyzed to extract turbine performance metrics.

To reduce data to useful form, free frame-by-frame video viewing software was utilized (VLC Media Player $\left.^{\mathrm{ix}}\right)$. The approximate data sampling rate was determined by placing a stopwatch in the video recorder's field of view and counting the number of frames shot over some characteristic

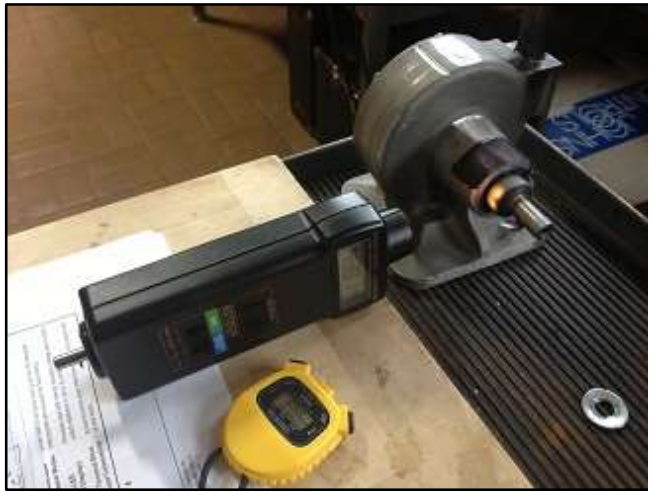

Figure 1: Optical tachometer and stopwatch positioned in the same video shot to enable video capture of turbine spindle experimental rotational velocity time histories for data analysis. 
duration. Each frame therefore showed the tachometer reading at a sampling interval equal to the frame rate of the video capture device used. An example of the entire set-up is shown in Figure 1.

\section{Turbine Rotational Inertia Determination}

The turbine was anchored about 2.5 meters above ground. One end of a long sewing thread was secured to the turbine spindle, and the other end was attached to a free weight of known mass resting at the elevation of the turbine. The thread was wrapped around the turbine shaft without doubling up on itself. With video capture of tachometer data enabled, the weight was knocked to the floor, spinning the turbine shaft with an instantaneous input of force provided by gravity acting on the weight's mass.

In general, turbine spindle angular acceleration under inlet gas pressure, $\ddot{\Theta}_{a}(t)$, under a falling mass, $\ddot{\phi}_{a}(t)$, or deceleration due to bearing friction, $\ddot{\Theta}_{d}(t)$, are approximated via the time derivative of collected experimental angular turbine data,

$$
\ddot{\Theta}(t)=\frac{\Delta \dot{\Theta}(t)}{\Delta t}
$$

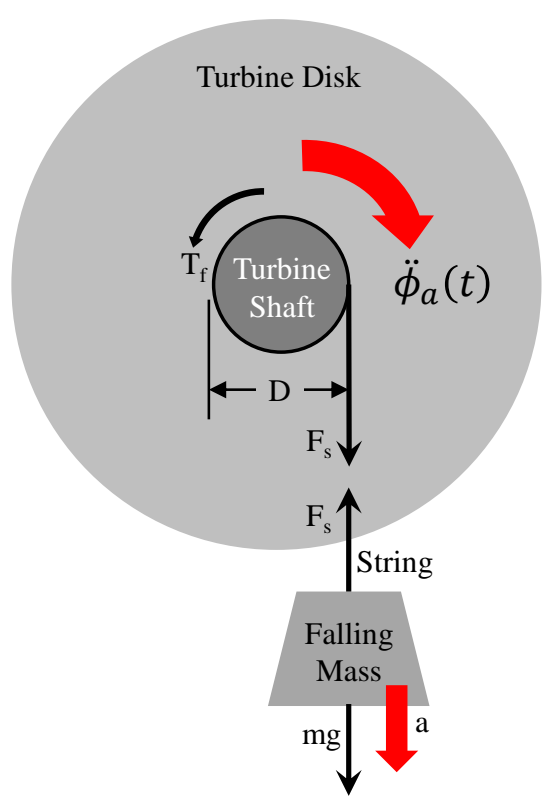

Figure 2: Free body diagram for weight-string-turbine system showing acceleration directions, forces, and torques acting on components.

where $\dot{\Theta}(t)$ is the spindle angular velocity, and $t$ is a time interval of measurement. Applying Newton's Second Law through a torque balance on the free body diagram in Figure 2, the difference between the torque imposed on the turbine shaft by the string, $F_{s}(D / 2)$, and the frictional torque from the bearings, $T_{f}$, upon which the turbine spindle is mounted is given by

$$
F_{s}\left(\frac{D}{2}\right)-T_{f}(t)=I \cdot \ddot{\phi}_{a}(t)
$$

where $I$ is the rotational inertia of the turbine spindle, $F_{s}$ is the force of the sting arising from the weight of the fixed mass, $m$, and $D$ is the diameter of the spindle around which the thread is wound. Finally, by applying a dynamic force balance to the falling mass alone (see Figure 2), the following expression results,

$$
m \cdot \ddot{\phi}_{a}(t) \cdot\left(\frac{D}{2}\right)=m \cdot g-F_{s}
$$

where $g$ is the local gravitational acceleration. This expression can be solved for $F_{s}$ :

$$
F_{s}=m \cdot g-m \cdot \ddot{\phi}_{a}(t) \cdot\left(\frac{D}{2}\right)
$$

To obtain an experimental function for $T_{f}$, the friction torque from the bearings, the unloaded turbine was spun up to its maximum operational rotational velocity using compressed air. When 
the turbine reached a steady state rate of rotation, the input gas was instantaneously shut off, and the rotational velocity of the turbine with respect to time was logged while the turbine spun down under friction primarily imposed by the bearings. A torque balance on the decelerating turbine spindle alone yields

$$
-T_{f}(t)=I \cdot \ddot{\Theta}_{d}(t)
$$

where $\ddot{\Theta}_{d}(t)$ is determined from Equation 1. Substituting Eq. 4 into Eq. 2 and rearranging gives

$$
I=\frac{F_{s}\left(\frac{D}{2}\right)}{\ddot{\phi}_{a}(t)-\ddot{\Theta}_{d}(t)}
$$

and plugging the $F_{s}$ expression of Equation $3 \mathrm{~b}$ into Equation 5 results in an expression to determine $I$ values exclusively from experimentally-measured inputs,

$$
I=\frac{\left[m g-m \ddot{\phi}_{a}(t) \frac{D}{2}\right]\left(\frac{D}{2}\right)}{\ddot{\phi}_{a}(t)-\ddot{\Theta}_{d}(t)}
$$

Now, to determine the value for $I$, the numerical values of the functions $\ddot{\phi}_{a}(t)$ and $\ddot{\Theta}_{d}(t)$ were found at each time step: $t_{1}, t_{2}, \ldots t_{n}$. These values were plugged into Equation 6 , which produced $\mathrm{n}-1$ values for $I$ where $\mathrm{n}$ is the total number of data points. The reported value for $I$ is the average of all the discrete $I$ values for each time step while the uncertainty in $I$ is approximated as twice the standard deviation of all the data.

\section{$\underline{\text { Turbine Power Curves }}$}

Turbine power output as a function of rotational velocity, the so-called turbine power curve, can be extracted experimentally by dynamic dynamometry. The turbine was run at 90 psi input pressure. Pressure upstream of the turbine was held constant using a regulator.

Video capture of tachometer data was initiated with the turbine at rest. The turbine gas inlet was instantaneously opened, and the turbine was allowed to spin up until its rotational velocity reached steady state (we describe later how 'steady state' is formally defined).

The following derivation leads to a turbine power curve expression. The turbine's moment of inertia, $I$, is already known from the above-described analysis. The turbine's power output, $P_{\text {out }}$, is

$$
P_{\text {out }}=T_{s} \cdot \dot{\Theta}_{a}(t)=I \cdot \frac{d \dot{\Theta}_{a}(t)}{\mathrm{d} t} \cdot \dot{\Theta}_{a}(t)
$$

since the output shaft torque, $T_{s}$, is

$$
T_{S}=I \cdot \frac{\mathrm{d} \dot{\Theta}_{a}(t)}{\mathrm{d} t}
$$

To find an equation for $P_{\text {out }}$, a functional form is needed for $\dot{\Theta}_{a}(t)$. This function can be determined by inspection. An example of a raw $\dot{\Theta}_{a}(t)$ data set is given in Figure 3 , and it is 
apparent from the non-zero initial slope that the function for $\dot{\Theta}_{a}(t)$ is a first order response (an asymptotic exponential) of the form

$$
\dot{\Theta}_{a}(t)=\dot{\Theta}_{\max } \cdot\left(1-\mathrm{e}^{-\frac{\mathrm{t}}{\tau}}\right)
$$

where $\dot{\Theta}_{\max }$ is the maximum turbine rotational velocity achieved at steady-state, and $\tau$ is a time constant characteristic of the system.

To fit Equation 9 to the experimental data and obtain a useful function for $\dot{\Theta}_{a}(t)$, the time constant, $\tau$, is treated as a variable parameter that is adjusted to achieve the best equation/experiment match. The fitting technique we used was minimization of the Standard Error of the Estimate (SEE). SEE is the sum of all the absolute differences between model and experiment at each

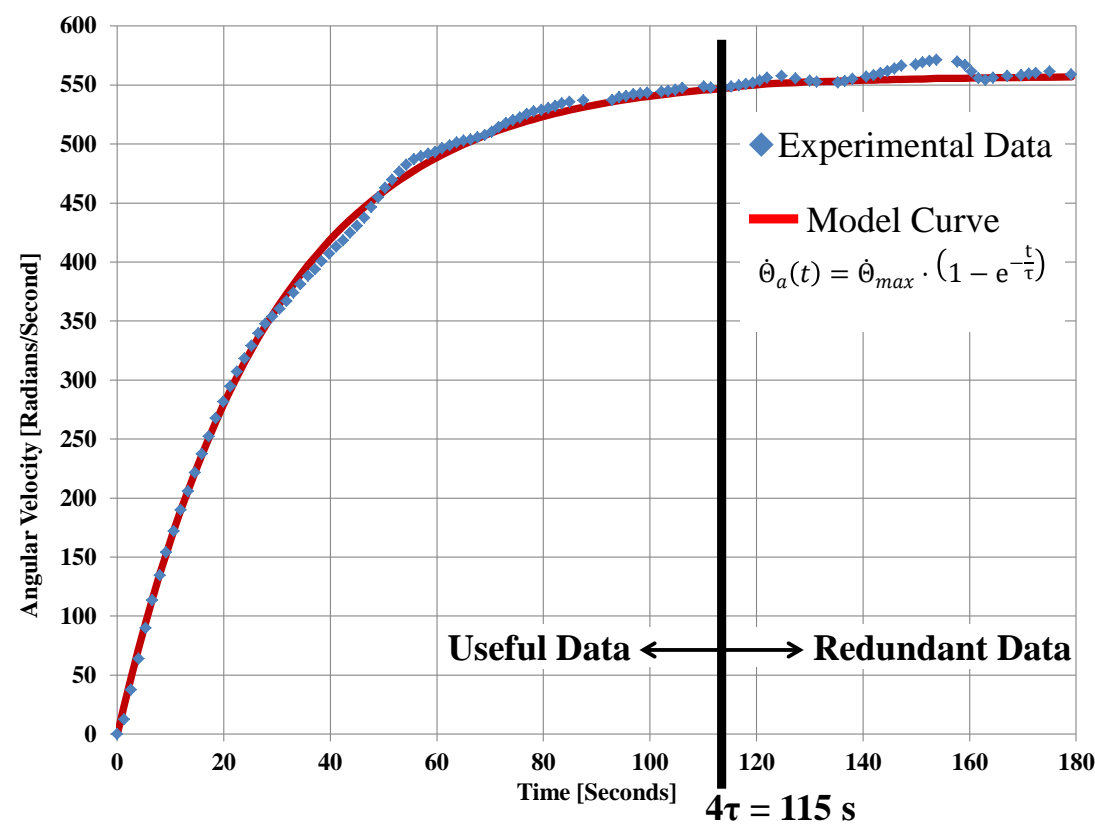

Figure 3: Rotational velocity versus time for a disk turbine spun up from rest at constant input gas pressure. The experimental data (blue diamonds) follow an asymptotic exponential, Equation 9, (red curve) where the system time constant, $\tau$, must be selected to provide the best data/model fit. Data collected after time $=4 \tau$ are redundant and can be eliminated from the analysis. discrete time step. As shown in Figure 3, exceptional experiment/model fit between real data and Equation 9 occurs when $\tau$ is correctly selected to minimize SEE.

Identifying the correct value for $\tau$ enables useful data reduction by approximating the time at which turbine steady state rotation rate was achieved. All data collected after this time can be discarded as redundant. We used $4 \tau$ as the number of time constants required for the system to reach steady state. This decision is justified via the following analysis. At time $=0, \dot{\Theta}_{a}(0)=0$. For a functional form of $\dot{\Theta}_{a}(t)=\dot{\Theta}_{\max }\left(1-\mathrm{e}^{-\frac{\mathrm{t}}{\tau}}\right)$, at time $=4 \tau, \dot{\Theta}_{a}(4 \tau)=\dot{\Theta}_{\max }\left(1-\mathrm{e}^{-\frac{4 \tau}{\tau}}\right) \approx$ $0.982 \cdot \dot{\Theta}_{\max }$. At time $\rightarrow \infty, \dot{\Theta}_{a}(\infty) \rightarrow \dot{\Theta}_{\max }$. Therefore, the percent error of $\dot{\Theta}_{a}(4 \tau)$ at time $=4 \tau$ relative to $\dot{\Theta}_{\text {max }}$ at time $\rightarrow \infty$ is given by the following calculation:

$$
\%=\frac{\dot{\Theta}_{\max } \dot{\Theta}_{a}(4 \tau)}{\dot{\Theta}_{\max }}=\frac{\dot{\Theta}_{\max }-(98.2 \%) \dot{\Theta}_{\max }}{\dot{\Theta}_{\max }} \approx 1.8 \%
$$

In other words, the percent error of $\dot{\Theta}_{a}(4 \tau)$ relative to $\dot{\Theta}_{\text {max }}$ is less than $2 \%$, which we deem to be an acceptable engineering approximation in characterizing this system. If additional error 
reduction is desired, data can be retained for a duration of $n \tau$ where $n$ is an arbitrary number selected based on the level of precision needed for calculations.

Given the experimentally determined functional form of $\dot{\Theta}_{a}(t)$ in Equation 9, the turbine output power, $P_{\text {out }}$, can be expressed by carrying out the derivative implied in Equation 7

$$
P_{\text {out }}=I \cdot \frac{d \dot{\Theta}_{a}(t)}{\mathrm{d} t} \cdot \dot{\Theta}_{a}(t)=I \frac{d\left[\dot{\Theta}_{\max }\left(1-\mathrm{e}^{-\frac{\mathrm{t}}{\tau}}\right)\right]}{d t} \dot{\Theta}_{a}(t)=\frac{I}{\tau} \cdot \dot{\Theta}_{a}(t) \cdot \dot{\Theta}_{\max } \cdot \mathrm{e}^{-\frac{\mathrm{t}}{\tau}}
$$

which reduces through the following algebraic manipulations to a second-order polynomial equation.

$$
\begin{gathered}
P_{\text {out }}=\frac{I}{\tau} \cdot \dot{\Theta}_{a}(t) \cdot\left[\dot{\Theta}_{\text {max }}-\dot{\Theta}_{\text {max }}+\dot{\Theta}_{\text {max }} \cdot \mathrm{e}^{-\frac{\mathrm{t}}{\tau}}\right] \\
P_{\text {out }}=\frac{I}{\tau} \cdot \dot{\Theta}_{a}(t) \cdot\left[\dot{\Theta}_{\text {max }}-\dot{\Theta}_{\text {max }} \cdot\left(1-\mathrm{e}^{-\frac{\mathrm{t}}{\tau}}\right)\right] \\
P_{\text {out }}=\frac{I}{\tau} \cdot \dot{\Theta}_{a}(t) \cdot\left[\dot{\Theta}_{\text {max }}-\dot{\Theta}_{a}(t)\right] \\
P_{\text {out }}=\frac{I}{\tau} \cdot\left[\dot{\Theta}_{\text {max }} \cdot \dot{\Theta}_{a}(t)-\dot{\Theta}_{a}(t)^{2}\right]
\end{gathered}
$$

For any compressed air input pressure, experimental values for $\tau$ and $\dot{\Theta}_{\text {max }}$ are determined using techniques described above.

\section{Discussion}

To evalaute how well the empirical power curve model of Equation 12d matches the turbine's actual performance turbine power data are directly extracted via dynamic dynamomtry by using an approximate differential form of Equation 7

$$
P_{\text {out }} \approx I \cdot \frac{\Delta \dot{\Theta}_{a}(t)}{\Delta t} \cdot \dot{\Theta}_{a}(t)
$$

Here, the differential rotational velocity measured during turbine spin-up at each time step substitutes for the pure derivative allowing the turbine power output at each rotational velocity to be

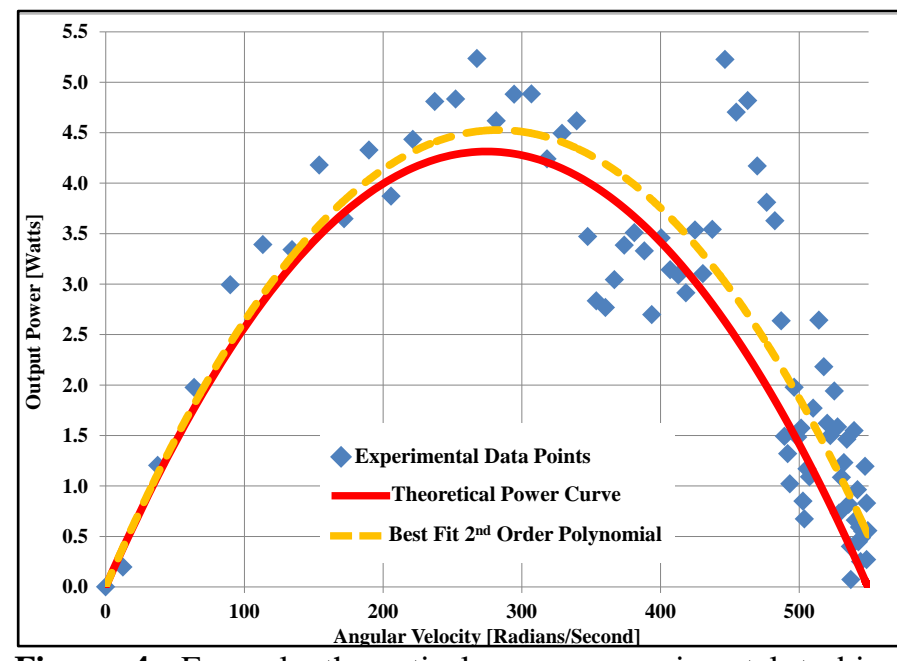

Figure 4: Example theoretical versus experimental turbine power curves obtained from dynamic dynamometry. The theoretical model slightly under-predicts peak power and the rotational velocity to achieve it compared to a polynomial fitted to experimental data. quantified. For 90 psi input pressure, the resulting comparision between the empirical power curve model of Equation $12 \mathrm{~d}$ and the discrete turbine power veruses rotational velocity represented by Eqauation 13 is shown in Figure 4. 
Of paramount importance to power system designers is the turbine's peak power and the rotational velocity the turbine must run at to achieve this maximum performance point. For the theoretical power curve, the maximum power point is found by setting the derivative of the function to zero,

$$
\begin{gathered}
\frac{d P_{\text {out }}}{d \dot{\Theta}_{a}(t)}=\frac{I}{\tau} \cdot\left[\dot{\Theta}_{\max }-2 \dot{\Theta}_{a}(t)\right]=0 \\
\dot{\Theta}_{a, \text { maxpower }}=\frac{\dot{\Theta}_{\text {max }}}{2}
\end{gathered}
$$

and maximum power is therefore

$$
\begin{gathered}
P_{\text {out }, \text { maxpower }}=\frac{I}{\tau} \cdot\left[\dot{\Theta}_{\text {max }} \cdot \frac{\dot{\Theta}_{\text {max }}}{2}-\left(\frac{\dot{\Theta}_{\text {max }}}{2}\right)^{2}\right] \\
P_{\text {out }, \text { maxpower }}=\frac{I}{\tau} \cdot\left[\frac{\left(\dot{\Theta}_{\text {max }}\right)^{2}}{4}\right]
\end{gathered}
$$

For the example case of 90 psi input pressure, $\dot{\Theta}_{\max }=549.55 \mathrm{rad} / \mathrm{sec}$. This measured result gives $\dot{\Theta}_{a \text {,maxpower }}=274.76 \mathrm{rad} / \mathrm{sec}$ for Eq. $14 \mathrm{~b}$ yielding $P_{\text {out } \text {,maxpower }}=4.32 \mathrm{watts}$ from Eq. $15 \mathrm{~b}$ for the theoretical power curve. By comparison, the curve fitted to the empirical data gives $\dot{\Theta}_{a, \text { maxpower }}=283.12 \mathrm{rad} / \mathrm{sec}$ yielding $P_{\text {out } \text { maxpower }}=4.52 \mathrm{watts}$. In other words, the theory under-predicts $\dot{\Theta}_{\text {a,maxpower }}$ by only $2.95 \%$ resulting in an underproduction of $P_{\text {out,maxpower }}$ by only $4.70 \%$. The predicative power of the theoretical model to pinpoint the turbine's maximum power point to better than $5 \%$ is an excellent and surprising result for such a simple analysis technique.

In addition to comparing the maximum power indicated by the theoretical curve of Eq. $12 \mathrm{~d}$ to the best-fit curve for all data, another metric to evaluate the predictive capacity of Eq. 12d is comparison to the actual maximum power directly measured via dynamic dynamometry. From experimental data, $P_{\text {out } \text { maxpower }}=5.23$ watts at $\dot{\Theta}_{a, \text { maxpower }}=267.70 \mathrm{rad} / \mathrm{sec}$. Thus, Eq. $15 \mathrm{~b}$ under-predicts maximum power by $17.5 \%$ and over-predicts the rotational velocity of the maximum power point by $2.58 \%$. However, since direct measurement of maximum power and maximum power point are based on discretely sampled data, it is possible that closer theory/experimental agreement could be achieved if the data were sampled at a higher rate.

\section{Conclusions}

For Rankine power cycles, the need to tightly regulate working fluid phase through the turbine limits the power produced and overall cycle efficiency. While Rankine cycles will someday dominate spacecraft power generation for manned space missions, limitations on power plant efficiency and power imposed by need for single-phase through-turbine flow will hinder adoption of this technology to power in interplanetary spacecraft. Disk turbines have the 
potential for continuous operation while processing two-phase (vapor-liquid) working fluid and are therefore a desirable technology to anchor space-based Rankine power cycles.

We describe a method called dynamic dynamometry to accurately measure and predict turbine mechanical power output using the rational inertia of the turbine's spinning components and friction in its bearings as the load. Using turbine spin-up, steady-state, and spin-down data captured via an optical tachometer, we present a method to indirectly determine the turbine's rotational inertia and predict its power curve. Comparing the resulting theoretical maximum power and rotational velocity for this point against a best fit curve for all experimental data yields excellent agreement. For the representative case of 90 psi turbine inlet pressure the maximum power is under-predicted by only $4.70 \%$ while the rotational velocity needed to operate at this point is under-predicted by only $2.95 \%$. We conclude that the derived theoretical model is a reasonable tool to analyze and predict the actual performance of disk turbines. This tool will be used for future experimental and theoretical evaluations of disk turbines for spacebased power generation using Rankine cycles.

\footnotetext{
${ }^{i}$ R. T. Lahey, V. Dhir, "Research in Support of the Use of Rankine Cycle Energy Conversion Systems for Space Power and Propulsion," Report NASA/CR-2004-213142, 2004.

ii T. Caillat, A. Borshchevsky, J. Fleurial, J. Snyder, "Space Technology and Applications International Forum," Editor: El-Genk, M. S., American Institute of Physics, 2000.

iii J. E. McKeathen, R. F. Reidy, S. K. S. Boetcher, M. J. Traum, “A Cryogenic Rankine Cycle for Space Power Generation," AIAA Paper Number 2009-4247, Proceedings of the 41st AIAA Thermophysics Conference, San Antonio, TX, June 22 - 25, 2009.

iv W. Rice, "Tesla Turbomachinery," In E. Logan \& R. Roy (Eds.), Handbook of Turbomachinery, Marcel Dekker, New York:, 2003, pp. 861-874.

${ }^{v}$ T. A. Emran, "Tesla Turbine Torque Modeling for Construction of a Dynamometer and Turbine," Masters Thesis, University of North Texas, 2011.

${ }^{v i}$ T. A. Emran, R. C. Alexander, C. T. Stallings, M. A. DeMay, M. J. Traum, "Method to Accurately Estimate Tesla Turbine Stall Torque for Dynamometer or Generator Load Selection," ASME Early Career Technical Journal, Volume 10, pp. 158-164, 2010 [URL: http://districts.asme.org/DistrictF/ECTC/2010ECTC.htm].

${ }^{v i i}$ V. G. Krishnan, Z. Iqbal, M. M. Maharbiz, "A micro Tesla turbine for power generation from low pressure heads and evaporation driven flows," Proceedings of the $16^{\text {th }}$ International Solid-State Sensors, Actuators and Microsystems Conference, Beijing, China, June 5-9, 2011, pp. 1851-1854.

viii Z. Yang, H. Weiss, M. J. Traum, "Gas Turbine Dynamic Dynamometry: A New Energy Engineering Laboratory Module," Proceedings of the 2013 ASEE North Midwest Section Conference, Fargo, North Dakota, Oct. 17-18, 2013.

${ }^{\text {ix } V C L ~ M e d i a ~ P l a y e r, ~ h t t p: / / w w w . v l c a p p . c o m / v l c-f e a t u r e s / f r a m e-b y-f r a m e-v i d e o-p l a y e r / ~, ~ a c c e s s e d ~ 7 / 30 / 2012 . ~}$
} 